\title{
A Non-Prenex, Non-Clausal QBF Solver with Game-State Learning
}

\author{
William Klieber, Samir Sapra, Sicun Gao, and Edmund Clarke* \\ Computer Science Department \\ Carnegie Mellon University \\ Pittsburgh, Pennsylvania
}

\begin{abstract}
We describe a DPLL-based solver for the problem of quantified boolean formulas (QBF) in non-prenex, non-CNF form. We make two contributions. First, we reformulate clause/cube learning, extending it to non-prenex instances. We call the resulting technique game-state learning. Second, we introduce a propagation technique using ghost literals that exploits the structure of a non-CNF instance in a manner that is symmetric between the universal and existential variables. Experimental results on the QBFLIB benchmarks indicate our approach outperforms other state-of-the-art solvers on certain benchmark families, including the tipfixpoint and tipdiam families of model checking problems.
\end{abstract}

Keywords: QBF, DPLL, non-clausal, non-prenex, clause learning

\section{Introduction}

Many problems in formal verification (among other areas) are naturally expressed in the language of QBF. Traditionally, QBF solvers have used conjunctive normal form $(\mathrm{CNF})$. Although CNF works well for SAT solvers, it hinders the work of QBF solvers by impeding the ability to detect and learn from satisfying assignments. In fact, a family of problems that are trivially satisfiable in negation-normal form (NNF) were experimentally found to require exponential time (in the problem size) for existing CNF solvers [18].

Various techniques have been proposed for avoiding the drawbacks of a CNF encoding. Zhang et al. have investigated dual CNF-DNF representations in which a boolean formula is transformed into a combination of an equi-satisfiable CNF formula and an equi-tautological DNF [18]. Sabharwal et al. have developed a QBF modeling approach based a game-theoretic view of QBF [14]. Ansotegui et al. have investigated the use of indicator variables [1]. These approaches all help to alleviate the problems of a pure CNF encoding, but we argue that a

\footnotetext{
^ This research was sponsored by the GSRC under contract no. 1041377 (Princeton University), National Science Foundation under contracts no. CCF0429120, no. CNS0926181, no. CCF0541245, and no. CNS0931985, Semiconductor Research Corporation under contract no. 2005TJ1366, General Motors under contract no. GMCMUCRLNV301, Air Force (Vanderbilt University) under contract no. 18727S3, International Collaboration for Advanced Security Technology of the National Science Council, Taiwan, under contract no. 1010717, and the Office of Naval Research under award no. N000141010188.
} 
fully non-clausal approach can lead to even greater improvements, especially for instances produced from deeply-nested circuits.

In addition to combined CNF-DNF techniques, fully non-clausal techniques have recently been investigated. A prenex circuit-based DPLL solver with "don't care" reasoning and clause/cube learning has been developed by Goultiaeva et al. [8]. A non-prenex NNF-based DPLL solver with dependency-directed (nonchronological) backtracking, but without learning, was developed by Egly, Seidl, and Woltran [4]. Non-clausal techniques using symbolic quantifier expansion (rather than DPLL) have been developed by Lonsing and Biere [10] and by Pigorsch and Scholl [13]. Giunchiglia et al. have developed a technique for miniscoping quantifiers (pushing quantifiers inward so as to minimize their scope) [7]. Non-clausal representations have also been investigated in the context of SAT solvers $[9,16,5]$.

Most existing DPLL-based QBF solvers perform clause/cube learning. However, traditional clause/cube learning was designed for prenex QBF instances, and it is not optimal for (or even directly applicable to) non-prenex QBF instances. We reformulate clause/cube learning and extend it to the non-prenex case. Additionally, we develop a new propagation technique using ghost literals. Experimental results indicate that our approach can beat other state-ofthe-art solvers on fixed-point computation instances of the type found in the tipfixpoint benchmark family.

\section{Preliminaries}

We consider non-prenex QBF formulas in negation-normal form ${ }^{1}$, as described by the following abstract grammar:

$$
\phi::=e_{i}\left|\neg e_{i}\right| u_{i}\left|\neg u_{i}\right| \phi \vee \ldots \vee \phi|\phi \wedge \ldots \wedge \phi| \exists e_{i} \phi \mid \forall u_{i} \phi
$$

We label each conjunction and disjunction with a gate variable of the form $g_{i}$, as illustrated in Figure 1. The conjunction/disjunction labelled $g_{i}$, together with its quantifier prefix (if any), is labelled with the primed gate variable $g_{i}^{\prime}$, as illustrated in Figure 1. As indicated in the abstract grammar, each labelled conjunction/disjunction may have any number of conjuncts/disjuncts.

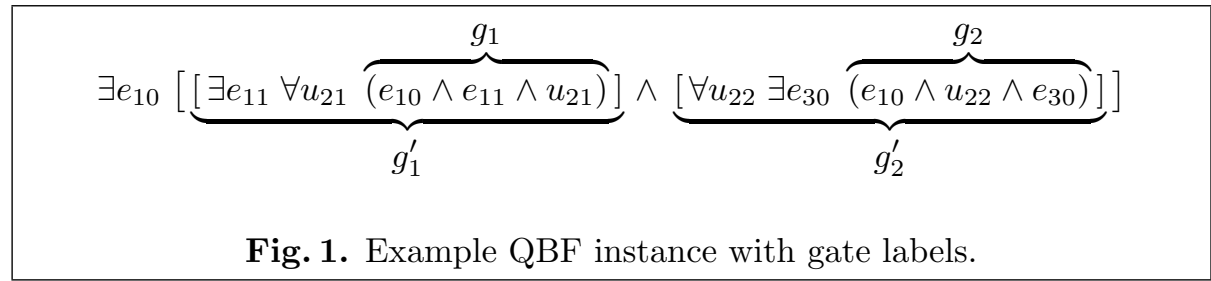

\footnotetext{
${ }^{1}$ Our solver does not require the use of strict NNF. Subformulas containing no quantifiers can be represented in circuit form.
} 
The term "gate variable" arises from the circuit representation of a propositional formula, in which a gate variable labels a logic gate.

Let "InFmla" denote the formula that the QBF solver is given as input. We impose the following restriction on InFmla: Every variable in InFmla must be quantified exactly once, and no variable may occur free (i.e., outside the scope of its quantifier). The variables that occur in InFmla are said to be input variables.

We represent an assignment $\pi$ by the set of literals assigned true by $\pi$. For example, the assignment $\left\{e_{1}, \neg u_{2}\right\}$ assigns $e_{1}$ true and assigns $u_{2}$ false, while leaving all other variables unassigned. We write " $\pi(\ell)$ " to denote the value (true, false, or undef) that $\pi$ assigns to $\ell$, as defined as follows: $\pi(\ell)=$ true if $\ell \in \pi, \pi(\ell)=$ false if $\neg \ell \in \pi$, and $\pi(\ell)=$ undef otherwise. For any variable $x$, we treat $\neg \neg x$ as equivalent to $x$. An assignment may not include both a variable and its negation. An input assignment is an assignment in which every assigned variable is an input variable (as opposed to a gate variable).

Definition 1 (Reduction). The reduction of a formula $f$ under an input assignment $\pi$, denoted by " $f \mid \pi$ ", is constructed from $f$ as follows: For each variable $x$ which is assigned a value by $\pi$, we delete the quantifier of $x$ and replace each occurrence of $x$ with its assigned value. For example, if $\pi=\left\{e_{1}\right\}$, then $\left[\exists e_{1} \cdot \forall u_{2} \cdot\left(e_{1} \wedge u_{2}\right)\right] \mid \pi=\left[\forall u_{2} \cdot\left(\right.\right.$ true $\left.\left.\wedge u_{2}\right)\right]$. Formally:

$$
\begin{aligned}
& \left.\ell\right|_{\pi}=\left\{\begin{array}{ll}
\pi(\ell) & \text { if } \pi(\ell) \neq \text { undef } \\
\ell & \text { if } \pi(\ell)=\text { undef }
\end{array} \quad(\exists x . f) \mid \pi= \begin{cases}\left.f\right|_{\pi} & \text { if } \pi(x) \neq \text { undef } \\
\exists x .(f \mid \pi) & \text { if } \pi(x)=\text { undef }\end{cases} \right. \\
& \left(f_{1} \wedge \ldots \wedge f_{n}\right) \mid \pi=\left(f_{1} \mid \pi\right) \wedge \ldots \wedge\left(f_{n} \mid \pi\right) \\
& \left(f_{1} \vee \ldots \vee f_{n}\right) \mid \pi=\left(f_{1} \mid \pi\right) \vee \ldots \vee\left(f_{n} \mid \pi\right)
\end{aligned} \quad(\forall x . f) \mid \pi= \begin{cases}\left.f\right|_{\pi} & \text { if } \pi(x) \neq \text { undef } \\
\forall x .(f \mid \pi) & \text { if } \pi(x)=\text { undef }\end{cases}
$$

Given two input literals $x$ and $y$, we say that $x$ is upstream of $y$ iff the scope of the quantifier of $x$ contains the quantifier of $y$. We say that a gate literal $g$ is upstream of an input literal $y$ iff every variable that occurs in the subformula $g$ is upstream of $y$.

\subsection{QBF as a Two-Player Game}

It is helpful to view QBF as a game between two players, Player E and Player U.

We make the following formal definitions:

- The existentially quantified variables are owned by Player E.

- The universally quantified variables are owned by Player U.

Informally, the game formulation goes as follows. Throughout the course of the game, the two players assign values to the variables that they own. The order in which the players assign variables is the quantification order of the variables. On each turn of the game, the owner of the outermost-quantified unassigned variable assigns it a value. The goal of Player $\mathrm{E}$ is to make InFmla true, and the goal of 
Player $\mathrm{U}$ is to make InFmla false. For non-prenex instances, we say that each quantifier-prefixed subformula (e.g., $g_{1}^{\prime}$ and $g_{2}^{\prime}$ in Figure 1) is a subgame. It may happen that two or more variables are quantified outermost; e.g., in Figure 1 on page 2 , after $e_{10}$ is assigned a value, both $e_{11}$ and $u_{22}$ are quantified outermost. In this case, two subgames have become independent of each other; they may be played in parallel or in series.

Definition 2 (Winning under an assignment). Player $\mathrm{U}$ wins a formula $f$ under $\pi$ iff $f \mid \pi$ is false. Player $\mathrm{E}$ wins a formula $f$ under $\pi$ iff $f \mid \pi$ is true. (See Definition 1 for the meaning of $f \mid \pi$.) (It would be more proper to say "has a winning strategy for" instead of "wins", but for brevity, we'll say simply "wins".)

For example, in Figure 1, Player $\mathrm{U}$ wins $g_{2}^{\prime}$ under the empty assignment, and Player E wins $g_{2}^{\prime}$ under $\left\{e_{10}\right.$ : true, $u_{22}$ : true $\}$.

Proposition 1 Player E wins $[\exists x \phi]$ under $\pi$ if he wins $\phi$ under either $\pi \cup\{x\}$ or $\pi \cup\{\neg x\}$. Player $\mathrm{U}$ wins $[\forall x \phi]$ under $\pi$ if he wins $\phi$ under either $\pi \cup\{x\}$ or $\pi \cup\{\neg x\}$.

\section{Symbolic Game States}

In this section, we introduce game-state learning, a reformulation of clause/cube learning. For prenex instances, the game-state formulation is isomorphic to clause/cube learning; the differences are merely cosmetic. However, the gamestate formulation is more convenient to extend to the non-prenex case.

To motivate the notation of game-state learning, we start by reviewing certain aspects of clause learning. Suppose the input formula InFmla is a prenex CNF QBF whose first clause is $\left(e_{1} \vee e_{3} \vee u_{4} \vee e_{5}\right)$. Under an assignment $\pi$, if all the literals in the clause are false, then clearly InFmla $\mid \pi$ is false. Moreover, if, under $\pi$, all the clause's existential literals are assigned false and none of the clause's universal literals are assigned true (i.e., they may either be assigned false or be unassigned), then InFmla $\mid \pi$ is false, since the universal player can win by making all the universal literals in the clause false.

As shown in [20], when the QBF clause learning algorithm is applied to

$$
\exists e_{1} \exists e_{3} \forall u_{4} \exists e_{5} \exists e_{7} .\left(e_{1} \vee e_{3} \vee u_{4} \vee e_{5}\right) \wedge\left(e_{1} \vee \neg e_{3} \vee \neg u_{4} \vee e_{7}\right) \wedge \ldots
$$

it can yield the tautological learned clause $\left(e_{1} \vee u_{4} \vee \neg u_{4} \vee e_{5} \vee e_{7}\right)$. Although counter-intuitive, this learned clause can be interpreted in the same way as a non-tautological clause: Under an assignment $\pi$, if all the clause's existential literals are assigned false and none of the clause's universal literals are assigned true, then InFmla $\mid \pi$ is false.

Learned cubes are similar: Under an assignment $\pi$, if all the cube's universal literals are assigned true and none of the cube's existential literals are assigned false, then InFmla| $\pi$ is true. With game-state learning, we explicitly separate the "must be true" literals from the "may be either true or unassigned" literals. (For non-prenex instances, the division is more complicated than just existential-vsuniversal.) Instead of writing a cube $\left(e_{1} \vee u_{2} \vee \neg e_{3}\right)$, we will write a game-state sequent $\left\langle\left\{u_{2}\right\},\left\{e_{1}, \neg e_{3}\right\}\right\rangle \models$ (E wins InFmla). 
Definition 3. A symbolic game state is a tuple $\left\langle L^{\text {now }}, L^{\text {fut }}\right\rangle$, where $L^{\text {now }}$ is a set of literals and $L^{\text {fut }}$ is a set of input literals. $\left\langle L^{\text {now }}, L^{\text {fut }}\right\rangle$ symbolically represents (or matches) exactly those input assignments under which:

1. every literal in $L^{\text {now }}$ reduces to true, and

2. no literal in $L^{\text {fut }}$ is assigned false - i.e., for every literal $\ell$ in $L^{\text {fut }}$, either $\ell$ is already true or $\ell$ has not yet been assigned a value (and therefore may become true in the future).

For example, consider again the QBF instance in Figure 1 on page 2. The assignment $\left\{\neg e_{10}\right\}$ matches both $\left\langle\left\{\neg g_{1}^{\prime}\right\}, \varnothing\right\rangle$ and $\left\langle\left\{\neg g_{1}^{\prime}\right\},\left\{u_{21}, \neg u_{21}\right\}\right\rangle$ (because $\neg e_{10}$ implies $\left.\neg g_{1}^{\prime}\right)$, but not $\left\langle\left\{\neg g_{1}^{\prime}\right\},\left\{e_{10}\right\}\right\rangle$. No assignment matches $\left\langle\left\{\neg e_{10}\right\},\left\{e_{10}\right\}\right\rangle$.

Definition 4 (Winning under a game state). We say that player $P$ wins a formula $f$ under a game state $G S$, written " $G S \models(P$ wins $f)$ ", iff $P$ wins $f$ under all assignments that match $G S$. Additionally, we say that $P$ loses $f$ under $G S$, written " $G S \models(P \operatorname{loses} f)$ ", iff the opponent of $P$ wins $f$ under $G S$.

For example, for the QBF instance in Figure 1:

- Neither player wins $g_{1}^{\prime}$ under the game state $\langle\varnothing, \varnothing\rangle$, because Player U loses under the matching assignment $\left\{e_{10}, e_{11}, u_{21}\right\}$ and Player $E$ loses under the matching assignment $\left\{\neg e_{10}\right\}$.

- Player U wins $g_{1}^{\prime}$ under $\left\langle\varnothing,\left\{\neg u_{21}\right\}\right\rangle$. For example, under the assignment $\pi=\left\{e_{11}\right\}, g_{1}^{\prime} \mid \pi$ is $\left[\forall u_{21}\left(e_{10} \wedge\right.\right.$ true $\left.\left.\wedge u_{21}\right)\right]$, which evaluates to false.

- Player E wins $g_{1}^{\prime}$ under $\left\langle\left\{u_{21}\right\},\left\{e_{10}, e_{11}\right\}\right\rangle$.

In our solver, instead of learning clauses or cubes, we maintain a game-state database with sequents of the form $G S \models\left(P\right.$ wins $\left.g_{i}^{\prime}\right)$. It turns out that whenever we learn a new game-state sequent for a prenex instance, the literals owned by the winner all go in $L^{\text {fut }}$, and the literals owned by the loser and the gate literals go in $L^{\text {now }}$. The relationship between learned game-state sequents and learned clauses/cubes (for prenex instances) is as follows. $\left\langle L^{\text {now }}, L^{\text {fut }}\right\rangle \models$ (U wins InFmla) is equivalent to the learned clause $\left[\neg \ell_{1} \vee \ldots \vee \neg \ell_{n}\right]$ where $\left\{\ell_{1}, \ldots, \ell_{n}\right\}=L^{\text {now }} \cup L^{\text {fut }}$ (where $L^{\text {now }}$ contains the loser/gate literals and $L^{\text {fut }}$ contains the winner literals). This equivalence is easily verified using the interpretation of learned clauses developed on the previous page. Likewise, $\left\langle L^{\text {now }}, L^{\text {fut }}\right\rangle \models$ (E wins InFmla) is equivalent to the learned cube $\left[\ell_{1} \wedge \ldots \wedge \ell_{n}\right]$ where $\left\{\ell_{1}, \ldots, \ell_{n}\right\}=L^{\text {now }} \cup L^{\text {fut }}$.

Proposition 2 If $\left\langle L^{\text {now }} \cup\{\ell\}, L^{\text {fut }}\right\rangle \models(P$ wins $f)$, and $\ell$ is owned by Player $P$ and the quantifier of $\ell$ is inside $f$, then $\left\langle L^{\text {now }}, L^{\text {fut }} \cup\{\ell\}\right\rangle \models(P$ wins $f)$, provided that $\neg \ell \notin L^{\text {fut }}$.

For example, consider the QBF instance $\forall u_{1} \cdot \exists e_{2} \cdot\left(u_{1} \oplus e_{2}\right)$, where " $u_{1} \oplus e_{2}$ " means " $\left(u_{1} \wedge \neg e_{2}\right) \vee\left(\neg u_{1} \wedge e_{2}\right)$ ". If Player E wins under $\left\langle\left\{u_{1}, \neg e_{2}\right\}, \varnothing\right\rangle$, then Proposition 2 tells us that Player E wins under $\left\langle\left\{u_{1}\right\},\left\{\neg e_{2}\right\}\right\rangle$. 


\section{Algorithm}

An overview of the top-level solver algorithm is provided in Figure 2. Initially, the current assignment CurAsgn is empty. For non-prenex instances, we may temporarily target in on a subgame of the input formula InFmla and ignore the rest; the subgame being targetted is recorded in the TargFmla global variable. On each iteration of the main loop, we first test to see if we know who wins TargFmla under the current assignment. There are two cases:

- If the winner of TargFmla is unknown, then we call DecideLit, which picks an unassigned input variable (from the first available quantifier block in the prefix of TargFmla) and assigns it a value in CurAsgn. If there are no more unassigned variables in the quantifier prefix of the current TargFmla, then we pick a new TargFmla from among the unassigned immediate subformulas of TargFmla and try again. After adding a new literal to CurAsgn, we call Propagate to perform boolean constraint propagation (BCP).

- If the winner is known, then we call LearnNewGS to learn a new gamestate sequent, adding it to the database. If the new game-state sequent reveals that InFmla evaluates to a value $v$ under the empty assignment, then we return $v$ as our final answer. Otherwise, we backtrack. We follow the well-known non-chronological backtracking technique, with the addition that we must also undo changes to TargFmla as appropriate. (That is, if we backtrack to the beginning of the $k^{\text {th }}$ decision level, then we must restore TargFmla to the value that it held at the beginning of the $k^{\text {th }}$ decision level. For this purpose, we maintain an array UndoTarg that maps each decision level to the value of TargFmla to be restored.) After backtracking, the newly-learned game-state sequent will force a literal, so we call Propagate to perform BCP. (Is a literal forced even when we leave a subgame $b$ by restoring an old value of TargFmla during backtracking? Yes; ghosts of $b$ are forced, as per case 1(b) in Section 4.3.)

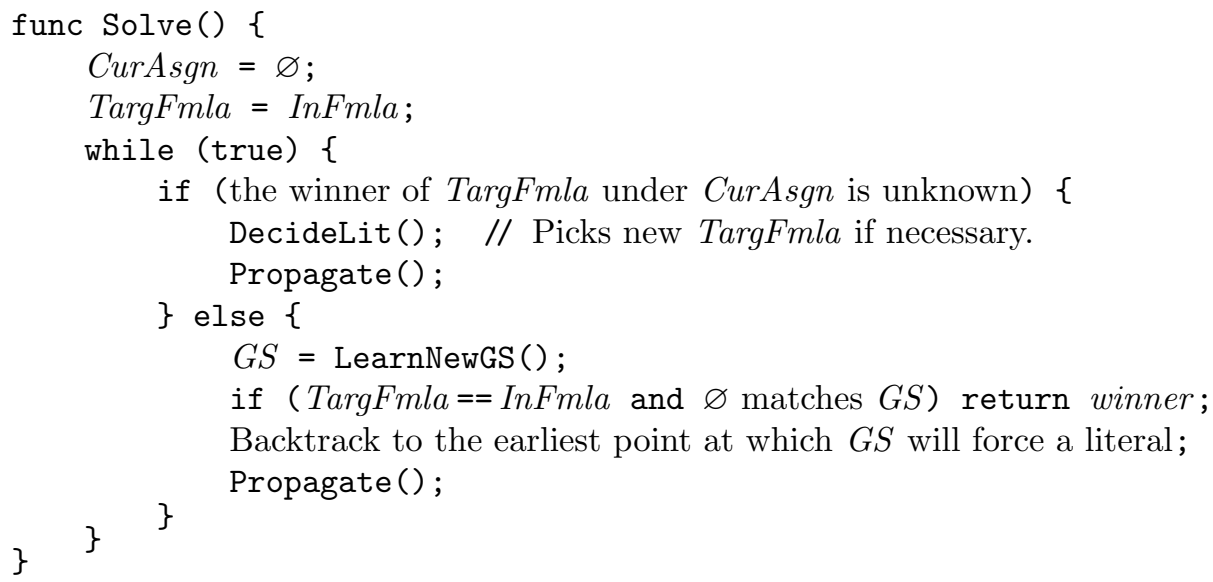

Fig. 2. Overview of top-level solver algorithm. 


\subsection{Ghost Literals}

Goultiaeva et al. [8] introduce a powerful propagation technique for QBF that significantly improves on existing QBF solvers on a variety of benchmarks. With their technique, if the solver notices that a gate literal $g$ must be true in order for the existential player to win, then $g$ becomes forced. However, this technique is asymmetric between the existential and universal players. A gate literal $g$ is forced if it is needed for the existential player to win, but not if it is needed for the universal player to win. We adapt this technique so that the universal variables benefit from the same propagation technique as do the existential variables and so that the learning procedure for satisfying assignments is just as powerful as for falsifying assignments.

In a prenex solver, for each gate variable $g$, we would introduce two ghost variables, $g\langle\mathrm{U}\rangle$ for Player $\mathrm{U}$ and $g\langle\mathrm{E}\rangle$ for Player E. A ghost literal $g\langle P\rangle$ would be forced whenever we detect that Player $P$ cannot win unless $g$ is made true.

For our non-prenex solver, we need to consider subgames (quantifier-prefixed subformulas, such as $g_{1}^{\prime}$ and $g_{2}^{\prime}$ in Figure 1). We introduce ghost variables of the form $g\langle\mathrm{U}, b\rangle$ and $g\langle\mathrm{E}, b\rangle$ where $b$ is a subgame which contains $g$ as a subformula. A ghost literal $g\langle P, b\rangle$ becomes forced when we detect that Player $P$ cannot win subgame $b$ without $g$ being true. For example, consider the below QBF instance (where $g_{1}$ is some propositional formula involving $e_{1}, u_{2}$, and $e_{3}$ ):

$$
\exists e_{1} \forall u_{2} \exists e_{3} \forall u_{4} \cdot[\underbrace{\left[\forall u_{5} \cdot g_{1} \vee u_{5}\right]}_{g_{2}^{\prime}} \wedge u_{4}] \vee \underbrace{\left[\forall u_{6} \cdot \neg g_{1} \vee u_{6}\right]}_{g_{3}^{\prime}}
$$

Under the empty assignment, $g_{1}\left\langle\mathrm{E}, g_{2}^{\prime}\right\rangle$ is forced (because Player E cannot win $g_{2}^{\prime}$ under $\varnothing$ unless $g_{1}$ is true) and likewise $\neg g_{1}\left\langle\mathrm{E}, g_{3}^{\prime}\right\rangle$ is forced.

In order to simplify the propagation and learning procedures, we allow game states to contain ghost literals. A game state with a ghost literal is said to match the same input assignments as if the game state contained the corresponding non-ghost gate literal; e.g., $\left\langle L^{\text {now }} \cup\{g\langle P, b\rangle\}, L^{\text {fut }}\right\rangle$ matches the same input assignments as $\left\langle L^{\text {now }} \cup\{g\}, L^{\text {fut }}\right\rangle$.

\subsection{Initialization of Game-State Database}

In CNF-based QBF solvers, the existential player owns the gate variables ${ }^{2}$, and there are clauses (generated from the Tseitin transformation [17]) that ensure that the existential player loses if he assigns a value to a gate variable that turns out to be inconsistent with the inputs to the gate. For example, if $g=e_{1} \wedge e_{2}$, then Player E would lose if he assigns $g=$ true and $e_{1}=$ false.

In our solver, instead of generating clauses via the Tseitin transformation, we generate game-state sequents. In a prenex solver, we would generate game-state sequents that ensure that a player $P$ loses if he assigns a ghost gate variable

\footnotetext{
${ }^{2}$ For CNF solvers, gate variables are introduced when formulas are converted to CNF via the Tseitin transformation [17]; these gate variables are existentially quantified.
} 
a value inconsistent with the gate's inputs. In our non-prenex solver, for each subgame $b$, we generate game-state sequents that ensure that a player $P$ loses subgame $b$ if he assigns a ghost gate variable $g\langle P, b\rangle$ a value inconsistent with the gate's inputs. For example, if $g=e_{1} \wedge e_{2}$ and subformula $g$ appears in a subgame $b$, then Player E would lose $b$ if he assigns $g\langle\mathrm{E}, b\rangle=$ true and $e_{1}=$ false. We construct such game-state sequents as follows. For every gate literal $g$, if $g$ labels a formula $\ell_{1} \wedge \ldots \wedge \ell_{n}$ (or $\neg g$ labels a formula $\neg \ell_{1} \vee \ldots \vee \neg \ell_{n}$ ), we add the following game-state sequents for each player $P \in\{\mathrm{E}, \mathrm{U}\}$ and each quantifierprefixed formula $b$ which contains $g$ as a subformula:

$-\left\langle\left\{\ell_{1}, \ldots, \ell_{n}, \neg g\right\}, \varnothing\right\rangle \models(P \operatorname{loses} b)$

$-\left\langle\left\{\neg \ell_{i}, g\right\}, \varnothing\right\rangle \models(P$ loses $b)$ for every $i \in\{1, \ldots, n\}$

For example, if $g_{3}=\neg e_{1} \vee \neg u_{2}$ and $g_{3}$ is a subformula of a subgame $g_{7}^{\prime}$, then we add game-state sequents $\left\langle\left\{e_{1}, u_{2}, g_{3}\right\}, \varnothing\right\rangle \models\left(\right.$ E loses $\left.g_{7}^{\prime}\right),\left\langle\left\{\neg e_{1}, \neg g_{3}\right\}, \varnothing\right\rangle \models$ (E loses $g_{7}^{\prime}$ ), and $\left\langle\left\{\neg u_{2}, \neg g_{3}\right\}, \varnothing\right\rangle \models$ (E loses $g_{7}^{\prime}$ ), among others.

After adding the game-state sequents to the database, we normalize them as follows. Consider a game-state sequent of the form $\left\langle L^{\text {now }}, L^{\text {fut }}\right\rangle=(P$ loses $b)$. First, we use Proposition 2 (on page 5) to move input literals owned by the winning player from $L^{\text {now }}$ to $L^{\text {fut }}$. Second, we replace each gate literal $g$ in $L^{\text {now }}$ with the ghost literal $g\langle P, b\rangle$. For example, consider a game-state sequent $\left\langle\left\{e_{1}, u_{2}, g_{3}\right\}, \varnothing\right\rangle \models\left(\right.$ E loses $\left.g_{7}^{\prime}\right)$. We move $u_{2}$ using Proposition 2 (assuming that the quantifier of $u_{2}$ is within the formula $\left.g_{7}^{\prime}\right)$ and replace $g_{3}$ with $g_{3}\left\langle\mathrm{E}, g_{7}^{\prime}\right\rangle$, yielding $\left\langle\left\{e_{1}, g_{3}\left\langle\mathrm{E}, g_{7}^{\prime}\right\rangle\right\},\left\{u_{2}\right\}\right\rangle \models\left(\mathrm{E}\right.$ loses $\left.g_{7}^{\prime}\right)$.

Recall that a ghost literal $g\langle P, b\rangle$ should become forced when $g$ must be true in order for $P$ to win $b$. Thus, for every quantifier-prefixed subformula $b$, the ghost literals $\neg b\langle\mathrm{U}, b\rangle$ and $b\langle\mathrm{E}, b\rangle$ should be forced. To ensure that the propagation procedure in Section 4.3 forces these literals, we add the following game-state sequents for every gate variable $b$ that labels a quantifier-prefixed formula:

$$
\begin{array}{ll}
-\langle\{b\langle\mathrm{U}, b\rangle\}, \varnothing\rangle \models(\mathrm{U} \text { loses } b) & (\text { to force } \neg b\langle\mathrm{U}, b\rangle) \\
-\langle\{\neg b\langle\mathrm{E}, b\rangle\}, \varnothing\rangle \models(\mathrm{E} \text { loses } b) & (\text { to force } b\langle\mathrm{E}, b\rangle)
\end{array}
$$

\subsection{Propagation and Forced Literals}

CurAsgn may contain forced ghost literals, so in general we can't say CurAsgn is a match for a game-state in the sense of Definition 3, because CurAsgn is not necessarily an input assignment. Instead, let us say that CurAsgn is a ghost match for a game-state sequent $\left\langle L^{\text {now }}, L^{\text {fut }}\right\rangle \models(P$ loses $b)$ iff every literal in $L^{\text {now }}$ is assigned true by CurAsgn and no literal in $L^{\text {fut }}$ is assigned false by CurAsgn.

During the Propagate procedure, conceptually we examine each learned game-state sequent $G S$ of the form $\left\langle L^{\text {now }}, L^{\text {fut }}\right\rangle \models(P$ loses $b)$ in which none of the literals in $L^{\text {now }} \cup L^{\text {fut }}$ are assigned false and $b$ is a subformula of TargFmla. There are three cases: 
1. If all literals in $L^{\text {now }}$ are true, then CurAsgn is a ghost match for $G S$, so $P$ loses $b$ under the current assignment. ${ }^{3}$ There are two subcases to consider:

(a) If $b=$ TargFmla, then we know who wins TargFmla under the current assignment, so we stop propagation and return to the Solve procedure.

(b) If $b \neq$ TargFmla, then for all subgames $s$ that contain $b$, the ghost variables $b\langle\mathrm{E}, s\rangle$ and $b\langle\mathrm{U}, s\rangle$ are forced to be false (if $P=\mathrm{E}$ ) or true (if $P=\mathrm{U}$ ).

2. If there is exactly one unassigned literal $\ell_{U}$ in $L^{\text {now }}$, then $\neg \ell_{U}$ is forced if:

(1) $\ell_{U}$ is owned by $P$ or is a ghost literal of the form $g\langle P, b\rangle$, and

(2) $\ell_{U}$ is upstream of all unassigned literals in $L^{\text {fut }}$, and

(3) $\ell_{U}$ does not appear outside subgame $b$ if $\ell_{U}$ is an input literal

(so that forcing $\neg \ell_{U}$ can't cause $P$ to lose a different subgame).

For example, consider again the QBF instance in Figure 1 on page 2. The game-state sequent $\left\langle\left\{u_{22}, \neg g_{2}\left\langle\mathrm{U}, g_{2}^{\prime}\right\rangle\right\},\left\{e_{10}, e_{30}\right\}\right\rangle \models\left(\mathrm{U}\right.$ loses $g_{2}^{\prime}$ ) will force $\neg u_{22}$ if CurAsgn $=\left\{\neg g_{2}\left\langle\mathrm{U}, g_{2}^{\prime}\right\rangle, e_{10}\right\}$. However, $\neg u_{22}$ will not be forced if CurAsgn $=\left\{\neg g_{2}\left\langle\mathrm{U}, g_{2}^{\prime}\right\rangle, e_{30}\right\}$, since $e_{10}$ is upstream of $u_{22}$, and thus Player $\mathrm{U}$ can delay assigning a value to $u_{22}$ until $E$ has assigned a value for $e_{10}$.

3. If more than one literal in $L^{\text {now }}$ is unassigned, then $G S$ doesn't force a literal.

When a game-state sequent $G S$ forces a literal $\ell$, we set antecedent $[\ell]=G S$.

Watched Literals. We use a straightforward adaptation of the watched-literals rule $[11,6]$. For each game-state sequent $\left\langle L^{\text {now }}, L^{\text {fut }}\right\rangle \models(P$ wins $g)$, we watch two literals in $L^{\text {now }}$ and one literal in $L^{\text {fut }}$.

Optimized Implementation of Ghost Literals. If a subformula $g$ occurs in a subgame $b$, and $b$ itself occurs in a larger subgame $s$, then we say that this occurrence of $g$ is an indirect occurrence in $s$. For example, in Figure $1, e_{10}$ occurs directly in $g_{1}^{\prime}$ and $g_{2}^{\prime}$ but occurs only indirectly in InFmla.

If a subformula $g$ occurs directly in only a single subgame $b$, then we only need to explicitly record only two ghost variables, $g\langle\mathrm{U}, b\rangle$ and $g\langle\mathrm{E}, b\rangle$. For any other quantified formula $s$ that contains $g$ as a subformula,

$$
\text { we infer } \underbrace{g\langle P, s\rangle \in C u r A s g n}_{(P \text { needs } g \text { to win } s)} \text { iff } \underbrace{g\langle P, b\rangle \in C u r A s g n}_{(P \text { needs } g \text { to win } b)} \text { and } \underbrace{b\langle P, s\rangle \in C u r A s g n}_{(P \text { needs } b \text { to win } s)}
$$

since the only way $g$ can influence the value of $s$ is via $b$. If a subformula $g$ occurs directly in multiple subgames, then we must record two ghost variables (existential and universal) for each subgame in which it directly occurs.

\footnotetext{
${ }^{3}$ Let $C u r A s g n_{I}=\{\ell \mid \ell \in$ CurAsgn and $\ell$ is an input literal $\}$. If all literals in $L^{\text {now }}$ are input literals, then $\operatorname{CurAsgn}_{I}$ matches $G S$, because all literals in $L^{\text {now }}$ are assigned true by $C u r A s g n_{I}$ and no literals in $L^{\text {fut }}$ are assigned false by $C u r A s g n_{I}$. If there are ghost literals in $L^{\text {now }}$, then $P$ is still doomed to lose $b$, because $P$ needs the corresponding gate literals to be true in order to win, but if these gate literals become true, then CurAsgn $_{I}$ will match $G S$ and $P$ loses under GS.
} 


\subsection{Learning New Game States}

As shown in Figure 2 on page 6 , when it becomes known which player wins TargFmla under the current assignment, we call LearnNewGS to learn a new game-state sequent. The only way for it to become known who wins TargFmla under CurAsgn is for CurAsgn to become a ghost match for a game-state sequent in the database (see case 1(a) in Section 4.3). Thus, when we enter LearnNewGS, the current assignment is a ghost match for some game state.

The procedure for learning a new game-state sequent is shown in Figure 3. We first make a copy of the existing game state that is a ghost match for the current assignment. We then remove the most recently forced literal in $L^{\text {now }}$ (not owned by the winner) by discharging it via its antecedent, as detailed in Figure 3. We continue to discharge until the $L^{\text {now }}$ slot either is empty or has a good unique implication point (UIP), as determined by the criteria from [19] ${ }^{4}$, or until we hit a literal quantified outside TargFmla.

For prenex instances, the procedure for discharging a forced literal is similar to resolution in clause learning: If $\left[x_{1} \vee \ldots \vee x_{n} \vee \ell\right]$ and $\left[\neg \ell \vee y_{1} \vee \ldots \vee y_{m}\right]$ are true, then $\left[x_{1} \vee \ldots \vee x_{n} \vee y_{1} \vee \ldots \vee y_{m}\right]$ is also true. The basic argument for the soundness of the discharge method goes as follows. Let $\left\langle L_{A}^{\text {now }} \cup\{\ell\}, L_{A}^{\text {fut }}\right\rangle \models(P$ wins $f)$ be $G S$, and let $\left\langle L_{B}^{\text {now }} \cup\{\neg \ell\}, L_{B}^{\text {fut }}\right\rangle \models(P$ wins $h)$ be the antecedent of $\ell$. Discharging $\ell$ via its antecedent yields $\left\langle L_{A}^{\text {now }} \cup L_{B}^{\text {now }}, L_{A}^{\text {fut }} \cup L_{B}^{\text {fut }}\right\rangle=(P$ wins $f)$. To simplify matters, let us assume that $\ell$ is upstream of every literal in $L_{B}^{\mathrm{fut}}$, so that $\ell$ is forced under any assignment that matches $\left\langle L_{B}^{\text {now }}, L_{B}^{\text {fut }}\right\rangle$. Since $P$ wins $f$ under any assignment that matches $\left\langle L_{A}^{\text {now }} \cup\{\ell\}, L_{A}^{\text {fut }}\right\rangle$, we conclude that if an assignment $\pi$ matches both $\left\langle L_{B}^{\text {now }}, L_{B}^{\text {fut }}\right\rangle$ and $\left\langle L_{A}^{\text {now }}, L_{A}^{\text {fut }}\right\rangle$ (i.e., if $\pi$ matches $\left\langle L_{A}^{\text {now }} \cup L_{B}^{\text {now }}, L_{A}^{\text {fut }} \cup L_{B}^{\text {fut }}\right\rangle$ ) then $\ell$ is forced and $P$ wins $f$.

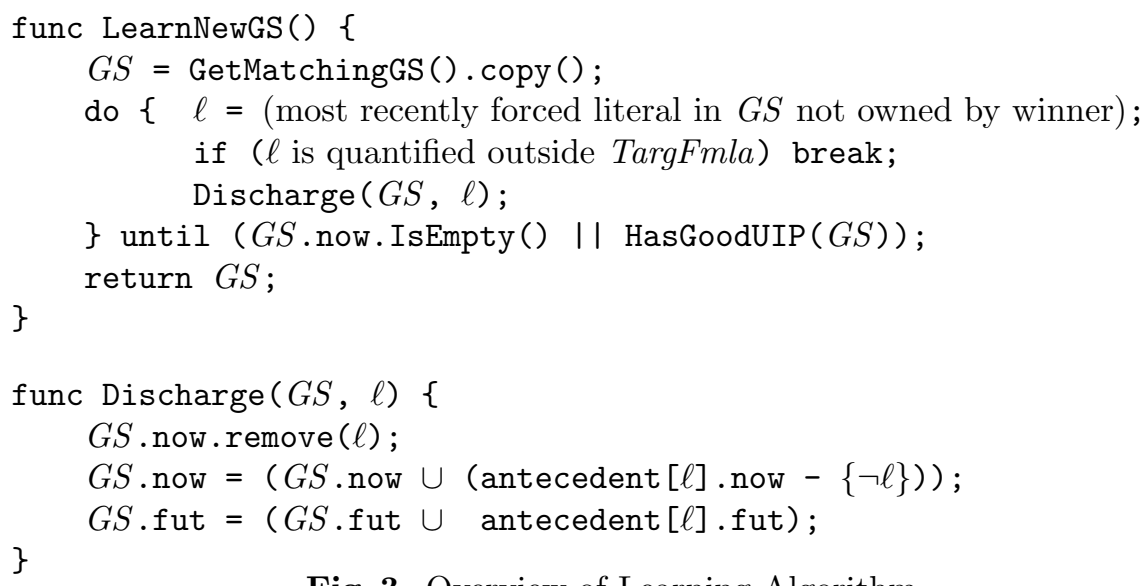

Fig. 3. Overview of Learning Algorithm

\footnotetext{
${ }^{4}$ Specifically, an input literal $\ell$ (owned by the loser) in $\left\langle L^{\text {now }}, L^{\text {fut }}\right\rangle$ is a good UIP if (1) the decision variable of $\ell$ 's decision level belongs to the losing player, (2) every literal in $\left(L^{\text {now }} \backslash\{\ell\}\right)$ belongs to an earlier decision level than $\ell$, and (3) every literal in $L^{\text {fut }}$ that is upstream of $\ell$ belongs to a decision level earlier than that of $\ell$.
} 
Example. Consider the QBF below.

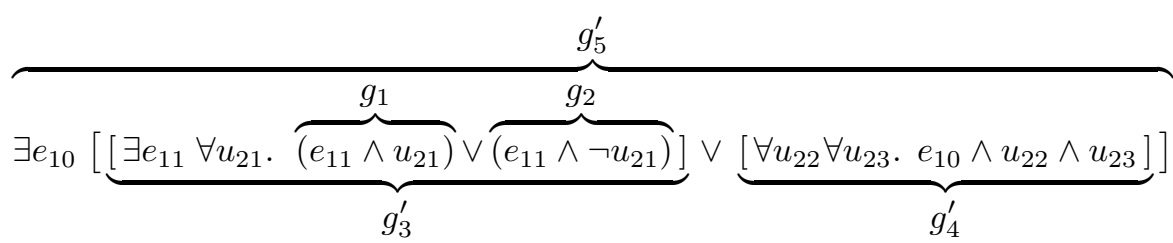

Fig. 4. Example non-prenex QBF instance

1. The initial assignment includes $g\left\langle\mathrm{E}, g^{\prime}\right\rangle$ and $\neg g\left\langle\mathrm{U}, g^{\prime}\right\rangle$ for $g \in\left\{g_{3}, g_{4}, g_{5}\right\}$.

2. $\left\langle\left\{g_{1}\left\langle\mathrm{U}, g_{3}^{\prime}\right\rangle, \neg g_{3}\left\langle\mathrm{U}, g_{3}^{\prime}\right\rangle\right\}, \varnothing\right\rangle \models$ (E wins $\left.g_{3}^{\prime}\right)$ forces $\neg g_{1}\left\langle\mathrm{U}, g_{3}^{\prime}\right\rangle$.

3. $\left\langle\left\{g_{2}\left\langle\mathrm{U}, g_{3}^{\prime}\right\rangle, \neg g_{3}\left\langle\mathrm{U}, g_{3}^{\prime}\right\rangle\right\}, \varnothing\right\rangle \models$ (E wins $\left.g_{3}^{\prime}\right)$ forces $\neg g_{2}\left\langle\mathrm{U}, g_{3}^{\prime}\right\rangle$.

4. Player E decides to assign $e_{10}=$ true.

5. All the variables in the outermost quantifier prefix are now assigned, so we must pick a subformula to investigate. We pick $g_{3}^{\prime}$ as the new target subformula.

6. Player E decides to assign $e_{11}=$ true.

7. $\left\langle\left\{u_{21}, \neg g_{1}\left\langle\mathrm{U}, g_{3}^{\prime}\right\rangle\right\},\left\{e_{11}\right\}\right\rangle \models\left(\mathrm{E}\right.$ wins $\left.g_{3}^{\prime}\right)$ forces $\neg u_{21}$.

8. $\left\langle\left\{\neg u_{21}, \neg g_{2}\left\langle\mathrm{U}, g_{3}^{\prime}\right\rangle\right\},\left\{e_{11}\right\}\right\rangle \models$ (E wins $g_{3}^{\prime}$ ) is a (ghost) match for the current assignment. Since $g_{3}^{\prime}$ is the current TargFmla, we learn a game state. We discharge $\neg u_{21}$, then $\neg g_{2}\left\langle\mathrm{U}, g_{3}^{\prime}\right\rangle$, then $\neg g_{1}\left\langle\mathrm{U}, g_{3}^{\prime}\right\rangle$, and finally $\neg g_{3}\left\langle\mathrm{U}, g_{3}^{\prime}\right\rangle$, yielding the new game-state sequent $\left\langle\varnothing,\left\{e_{11}\right\}\right\rangle \models\left(\mathrm{E}\right.$ wins $\left.g_{3}^{\prime}\right)$.

9. We now backtrack, removing $e_{11}$ and $e_{10}$ from the current assignment and reverting TargFmla to InFmla.

10. Having backtracked, our newly learned game-state sequent now forces $g_{3}\left\langle\mathrm{U}, g_{5}^{\prime}\right\rangle$.

11. $\left\langle\left\{g_{3}\left\langle\mathrm{U}, g_{5}^{\prime}\right\rangle, \neg g_{5}\left\langle\mathrm{U}, g_{5}^{\prime}\right\rangle\right\}, \varnothing\right\rangle \models$ (E wins InFmla) matches current assignment.

12. We learn the new game-state sequent $\left\langle\varnothing,\left\{e_{11}\right\}\right\rangle=(\mathrm{E}$ wins InFmla).

13. The empty assignment matches this new game-state, so our final answer is that InFmla $=$ true.

\section{Experimental Results}

We implemented the ideas in this paper in a solver which we call GhostQ. In our experimental results, GhostQ always did at least as well as CirQit and it outperformed Qube on the k, tipdiam, and tipfixpoint families.

We ran GhostQ on the non-CNF instances from QBFLIB on $2.66 \mathrm{GHz}$ machine with a timeout of 300 seconds. For comparison we show the results for CirQit published in [8] (which were conducted on a $2.8 \mathrm{GHz}$ machine with a timeout of 1200 seconds). (CirQit is not publicly available.) As shown in Table 1, GhostQ performs better CirQit on every benchmark family except consistency. 
Table 1. Comparison between GhostQ and CirQit.

\begin{tabular}{|lr|rr|rr|}
\hline Family & inst. & \multicolumn{2}{|c|}{ GhostQ } & \multicolumn{2}{|c|}{ CirQit } \\
\hline Seidl & 150 & $\mathbf{1 5 0}$ & $(1606 \mathrm{~s})$ & 147 & $(2281 \mathrm{~s})$ \\
assertion & 120 & $\mathbf{1 2}$ & $(141 \mathrm{~s})$ & 3 & $(1 \mathrm{~s})$ \\
consistency & 10 & 0 & $(0 \mathrm{~s})$ & 0 & $(0 \mathrm{~s})$ \\
counter & 45 & $\mathbf{4 0}$ & $(370 \mathrm{~s})$ & 39 & $(1315 \mathrm{~s})$ \\
dme & 11 & $\mathbf{1 1}$ & $(13 \mathrm{~s})$ & 10 & $(15 \mathrm{~s})$ \\
possibility & 120 & $\mathbf{1 4}$ & $(274 \mathrm{~s})$ & 10 & $(1707 \mathrm{~s})$ \\
ring & 20 & $\mathbf{1 8}$ & $(28 \mathrm{~s})$ & 15 & $(60 \mathrm{~s})$ \\
semaphore & 16 & $\mathbf{1 6}$ & $(4 \mathrm{~s})$ & 16 & $(7 \mathrm{~s})$ \\
\hline Total & 492 & 261 & $(2435 \mathrm{~s})$ & 240 & $(5389 \mathrm{~s})$ \\
\hline
\end{tabular}

Table 2. Comparison between GhostQ and Qube.

\begin{tabular}{|lr|rr|rr|}
\hline Family & inst. & \multicolumn{2}{|c|}{ GhostQ } & \multicolumn{2}{|c|}{ Qube } \\
\hline bbox-01x & 450 & 171 & $(133 \mathrm{~s})$ & $\mathbf{3 4 1}$ & $(1192 \mathrm{~s})$ \\
bbox_design & 28 & 19 & $(256 \mathrm{~s})$ & $\mathbf{2 8}$ & $(15 \mathrm{~s})$ \\
bmc & 132 & 43 & $(266 \mathrm{~s})$ & $\mathbf{4 9}$ & $(239 \mathrm{~s})$ \\
$\mathrm{k}$ & 61 & $\mathbf{4 2}$ & $(355 \mathrm{~s})$ & 13 & $(55 \mathrm{~s})$ \\
$\mathrm{s}$ & 10 & 10 & $(1 \mathrm{~s})$ & 10 & $(5 \mathrm{~s})$ \\
tipdiam & 85 & $\mathbf{7 2}$ & $(143 \mathrm{~s})$ & 60 & $(235 \mathrm{~s})$ \\
tipfixpoint & 196 & $\mathbf{1 6 5}$ & $(503 \mathrm{~s})$ & 100 & $(543 \mathrm{~s})$ \\
sort_net & 53 & 0 & $(0 \mathrm{~s})$ & $\mathbf{1 9}$ & $(176 \mathrm{~s})$ \\
all other & 121 & 9 & $(38 \mathrm{~s})$ & $\mathbf{2 3}$ & $(227 \mathrm{~s})$ \\
\hline Total & 1136 & $531(1695 \mathrm{~s})$ & $643(2687 \mathrm{~s})$ \\
\hline
\end{tabular}

Table 3. Comparison between GhostQ and Non-DPLL Solvers.

\begin{tabular}{|lr|r|r|r|r|r|}
\hline & & \multicolumn{2}{|c|}{ Timeout $60 \mathrm{~s}$} & \multicolumn{2}{c|}{ Timeout 600 s } \\
\hline Family & inst. & GhostQ & Quantor & sKizzo & GhostQ & AIGsolve \\
\hline bbox-01x & 450 & 171 & 130 & 166 & 178 & 173 \\
bbox_design & 28 & 19 & 0 & 0 & 22 & 23 \\
bmc & 132 & 43 & 106 & 83 & 51 & 30 \\
$\mathrm{k}$ & 61 & 42 & 37 & 47 & 51 & 56 \\
S & 10 & 10 & 8 & 8 & 10 & 10 \\
tipdiam & 85 & 72 & 23 & 35 & 72 & 77 \\
tipfixpoint & 196 & 165 & 8 & 25 & 170 & 133 \\
sort_net & 53 & 0 & 27 & 1 & 0 & 0 \\
all other & 121 & 9 & 49 & 31 & 17 & 35 \\
\hline Total & 1136 & 531 & 388 & 396 & 571 & 537 \\
\hline
\end{tabular}

In Tables 1-2, we give the number of instances solved and the time needed to solve them. (Times shown do not include time spent trying to solve instances where the solver timed out.) In Table 3, we give the number of instances solved. 
The ring and semaphore families consist of prenex instances. The other families are non-prenex, so our solver took advantage of its ability to perform non-prenex game-state learning. During testing of our solver, it was noted that non-prenex learning was especially helpful on the dme family. ${ }^{5}$

We compared GhostQ to the state-of-the-art solvers Qube 6.6 [7], Quantor 3.0 [3], and sKizzo 0.8.2 [2]. We ran these solvers on the QBFLIB QBFEVAL 2007 benchmarks [12] on a $2.66 \mathrm{GHz}$ machine, with a time limit of 60 seconds and a memory limit of $1 \mathrm{~GB}$. The results are shown in Tables 2 and 3. We also show the results for AIGsolve published in [13], but these numbers are not directly comparable because they were obtained on a different machine and with a timeout of 600 seconds.

For the CNF benchmarks, we wrote a script to reverse-engineer the QDIMACS file to circuit form and convert it to our solver's input format. (This is similar to the technique in [13], but we also looked for "if-then-else" gates of the form $g=(x ? y: z)$.) Of the four other solvers shown in Tables 2 and 3, Qube is the only other DPLL-based solver, so it is most similar to our solver. Our experimental results show that GhostQ does better than Qube on the tipdiam and tipfixpoint families (which concern diameter and fixpoint calculations for model checking problems on the TIP benchmarks) and on the $\mathrm{k}$ family.

The use of ghost literals can help GhostQ in two ways: (1) By treating the gate literals specially instead of treating them as belonging to the existential player, we can more readily detect satisfactions and we can learn more powerful cubes; (2) By using universal ghost literals, we have a more powerful propagation procedure for the universal input literals. (We did not perform unprenexing on any of the originally-CNF benchmarks, so our use of game-state learning doesn't improve performance here.) To further investigate, we turned off downward propagation of universal ghost literals; on most families the effect was negligible, but on tipfixpoint we solved only 149 instances instead of 165 .

\section{Conclusion}

In this paper, we have made two contributions. First, we have introduced the concept of symbolic game states and used this concept to reformulate clause/cube learning and extend it to the non-prenex case. Using game states, we have also been able to reformulate the techniques for conflict/satisfaction analysis, BCP, and non-chronological backtracking. In all cases, we give a unified presentation which is applicable to both the existential and universal players, instead of using separate terminology and notation for the two players. Further, game states are 'well-behaved' theoretically, in that we no longer need learn and store tautological clauses (or contradictory cubes). Our second contribution is introducing the concept of ghost literals, allowing us to improve upon the propagation technique introduced in [8] by eliminating the asymmetry between the players so that

\footnotetext{
${ }^{5}$ The dme family instances were originally given in prenex form, but we pushed the quantifiers inward as a preprocessing step. The unprenexing time was about 0.8 seconds per instance and is included in our solver's total time shown in the table.
} 
the technique can reduce the search space for both the universal and existential players (instead of only the existential player). Experiments show that our techniques work particularly well on certain benchmarks related to formal verification. For future work, it may be worthwhile to investigate whether the ideas of dynamic partitioning [15] can be extended to allow dynamic unprenexing.

\section{References}

1. C. Ansótegui, C. P. Gomes, and B. Selman. The Achilles' Heel of QBF. In AAAI 2005.

2. M. Benedetti. Evaluating QBFs via Symbolic Skolemization. In LPAR 2004.

3. A. Biere. Resolve and Expand. In SAT 2004.

4. U. Egly, M. Seidl, and S. Woltran. A Solver for QBFs in Nonprenex Form. In ECAI 2006 .

5. M. K. Ganai, P. Ashar, A. Gupta, L. Zhang, and S. Malik. Combining strengths of circuit-based and CNF-based algorithms for a high-performance SAT solver. In DAC 2002.

6. I. P. Gent, E. Giunchiglia, M. Narizzano, A. G. D. Rowley, and A. Tacchella. Watched Data Structures for QBF Solvers. In SAT 2003.

7. E. Giunchiglia, M. Narizzano, and A. Tacchella. Quantifier structure in search based procedures for QBFs. In DATE 2006.

8. A. Goultiaeva, V. Iverson, and F. Bacchus. Beyond CNF: A Circuit-Based QBF Solver. In SAT 2009.

9. H. Jain, C. Bartzis, and E. M. Clarke. Satisfiability Checking of Non-clausal Formulas Using General Matings. In SAT 2006.

10. F. Lonsing and A. Biere. Nenofex: Expanding NNF for QBF Solving. In SAT 2008.

11. M. W. Moskewicz, C. F. Madigan, Y. Zhao, L. Zhang, and S. Malik. Chaff: Engineering an Efficient SAT Solver. In DAC 2001.

12. M. Narizzano, L. Pulina, and A. Tacchella. QBFEVAL. http://www.qbfeval.org/.

13. F. Pigorsch and C. Scholl. Exploiting structure in an AIG based QBF solver. In DATE 2009.

14. A. Sabharwal, C. Ansótegui, C. P. Gomes, J. W. Hart, and B. Selman. QBF Modeling: Exploiting Player Symmetry for Simplicity and Efficiency. In SAT 2006.

15. H. Samulowitz and F. Bacchus. Dynamically Partitioning for Solving QBF. In SAT $200 \%$.

16. C. Thiffault, F. Bacchus, and T. Walsh. Solving Non-clausal Formulas with DPLL Search. In Constraint Programming - CP 2004.

17. G. S. Tseitin. On the complexity of derivations in the propositional calculus. Studies in Constructive Mathematics and Mathematical Logic, Part II, ed. A.O. Slisenko, 1968.

18. L. Zhang. Solving QBF by Combining Conjunctive and Disjunctive Normal Forms. In AAAI 2006.

19. L. Zhang and S. Malik. Towards a Symmetric Treatment of Satisfaction and Conflicts in Quantified Boolean Formula Evaluation. In Constraint Programming - CP 2002.

20. L. Zhang and S. Malik. Conflict Driven Learning in a Quantified Boolean Satisfiability Solver. In ICCAD 2002, 2002. 